\title{
The nuclear fragmentation problem and Bormio's contribution to its solution
}

\author{
Wolfgang Bauer ${ }^{1}$ \\ Department of Physics and Astronomy, Michigan State University and \\ National Superconducting Cyclotron Laboratory \\ 567 Wilson Road, Room 4208 \\ East Lansing, MI 48824 \\ USA \\ E-mail: bauerepa.msu.edu
}

I examine the progress made in the theoretical understanding of nuclear multi-fragmentation during the previous quarter century. In particular, I examine the evidence that nuclear fragmentation events are connected with a phase transition of nuclear matter in finite systems.

50th International Winter Meeting on Nuclear Physics,

Bormio, Italy

23-27 January 2012

$1 \quad$ Speaker 


\section{Introduction: Equation of State}

The nuclear force is repulsive at short distances and attractive at intermediate distances; in this respect it is just like a Van der Waals force. In equilibrium thermodynamics one can find relations between the state variables temperature, pressure, and volume and express these as an equation of state for a given force.
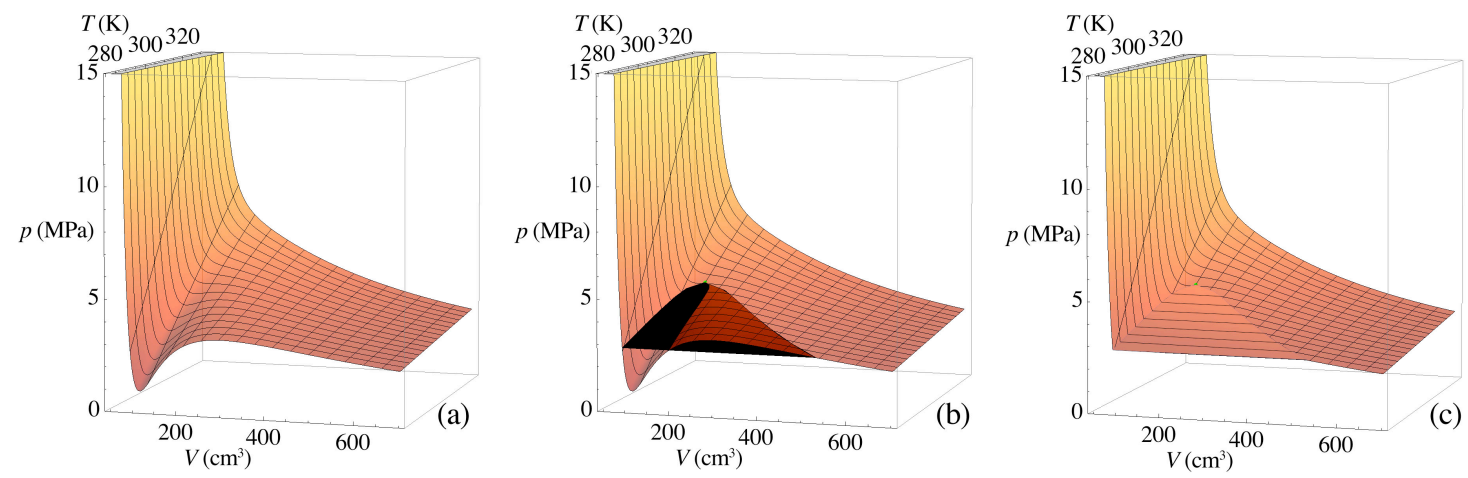

Figure 1: Equation of state for 1 mole of ethane, $\mathrm{C}_{2} \mathrm{H}_{6}$ : (a) Van der Waals equation of state, (b) Maxwell construction, (c) real equation of state for ethane. [1]

Figure 1 shows an example for the Van der Waals like equation of state of ethane, $\mathrm{C}_{2} \mathrm{H}_{6}$. Invariably, Van der Waals type forces lead to a transition between the high-temperature gas like phase and a low-temperature liquid phase. In part (a) of Figure 1 the equation of state $f(T, p, V)$ shows oscillations for low values of the temperature, which are characteristic. In part (b) these are removed via a Maxwell construction, which lead to horizontal isotherms (constant pressure) in the coexistence region of the gas and the liquid phase. The coexistence region terminates at the critical temperature, at which this first order phase transition becomes continuous. For the last 3-4 decades, it has been one of the main goals to find evidence for a similar phase diagram for nuclear matter [2].

Over the last few decades we have accumulated a large number of datasets with promising hints on the existence of a first order phase transition [3] and scale-invariant power-law spectra near the critical point [4].

\section{Non-Equilibrium Phase Transitions}

\subsection{Problems with an equilibrium thermodynamics framework}

If the goal is to find evidence of phase coexistence between a nuclear quantum liquid and a hadron gas, or even to find the critical point and to determine the critical exponents, then one has to ask if the conventional assumptions for studying an equilibrium phase transition in the laboratory are fulfilled. Since the typical dimensions in space $\left(\sim 10^{-14} \mathrm{~m}\right)$ and time $\left(\sim 10^{-21} \mathrm{~s}\right)$ of a high-energy heavy ion collision are too small for direct observation, we can only observe 
asymptotic momentum states of the reaction products. While many ingenious contributions have been made towards measuring the state variables under these limiting conditions, it is clear that a heavy ion collision does not allow us to make individual measurements at a unique point in $(T, p, V)$ space. Instead any individual collision represents a path through this space from the initial to the final point. Furthermore, it is not even clear that thermodynamic equilibrium can be established. In particular near the critical point numerical simulations establish the existence of critical slowing down, i.e. a slower and slower approach to equilibrium the closer the state of the system approaches the location of the critical point. The appearance of power laws in experimental data should therefore be a rare event, not a ubiquitous one. But power laws, appropriately modified for finite-size cutoffs, seem to appear everywhere in nuclear fragmentation data.

\subsection{Self-Organized Critical Systems}

An interesting alternative way to obtaining power laws in nature is through metastable systems that are driven to scale-invariant distributions via sequences of "avalanches" resulting from the interplay of stress and relief. Perhaps the most famous example of this is the sand pile model advanced by Bak and collaborators [5,6]. Scale-invariant power-law distributions, exponential cut-offs due to finite sizes, as well as connections to diffusion physics and limits of predictability can be found in these systems, some of them as simple as word-wrap cascades in modern word processors [7].

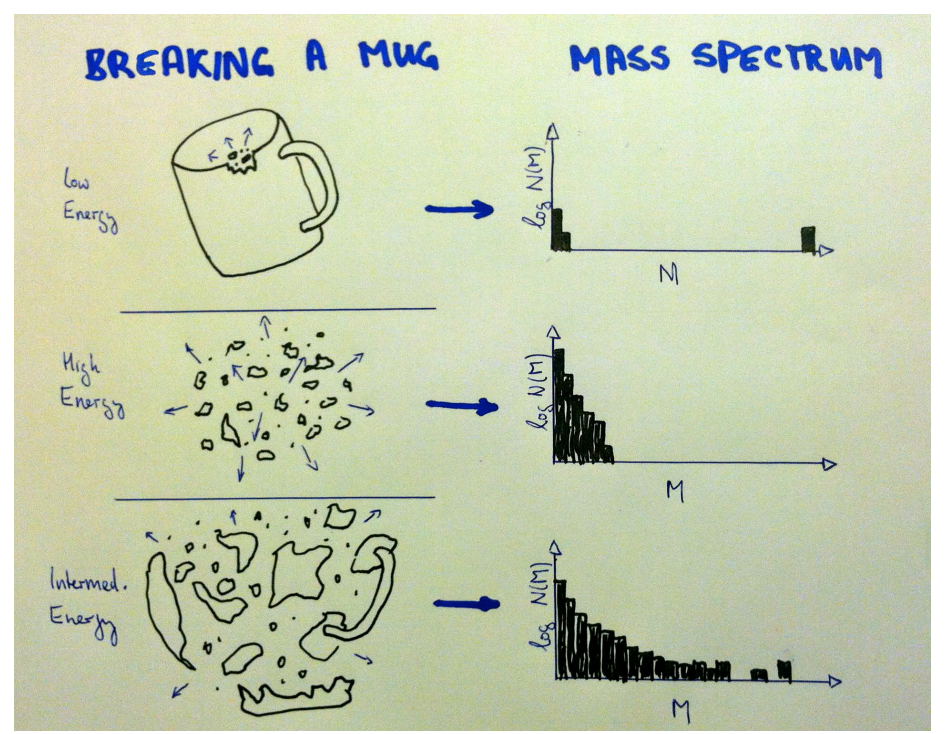

Figure 2: The analogy of nuclear fragmentation with the breaking of a coffee mug, as introduced by us at the 1985 Bormio meeting.

One system that falls into this category of self-organized non-equilibrium phase transitions is the shattering of coffee mugs, a model we introduced at the 1985 Bormio meeting [8] (see Figure 2). This model predicts exponentially falling mass spectra in the low-to-medium mass regime, with long power-law distributions at intermediate energies. As shown in Figure 3, one 
can even build an "accelerator" for coffee mugs and perform this type of fragmentation studies in experimental fashion. High-speed photography enables us to analyze the time evolution of these fragmentation events and even shows evidence for the formation of a shockwave (right frame of Figure 3).
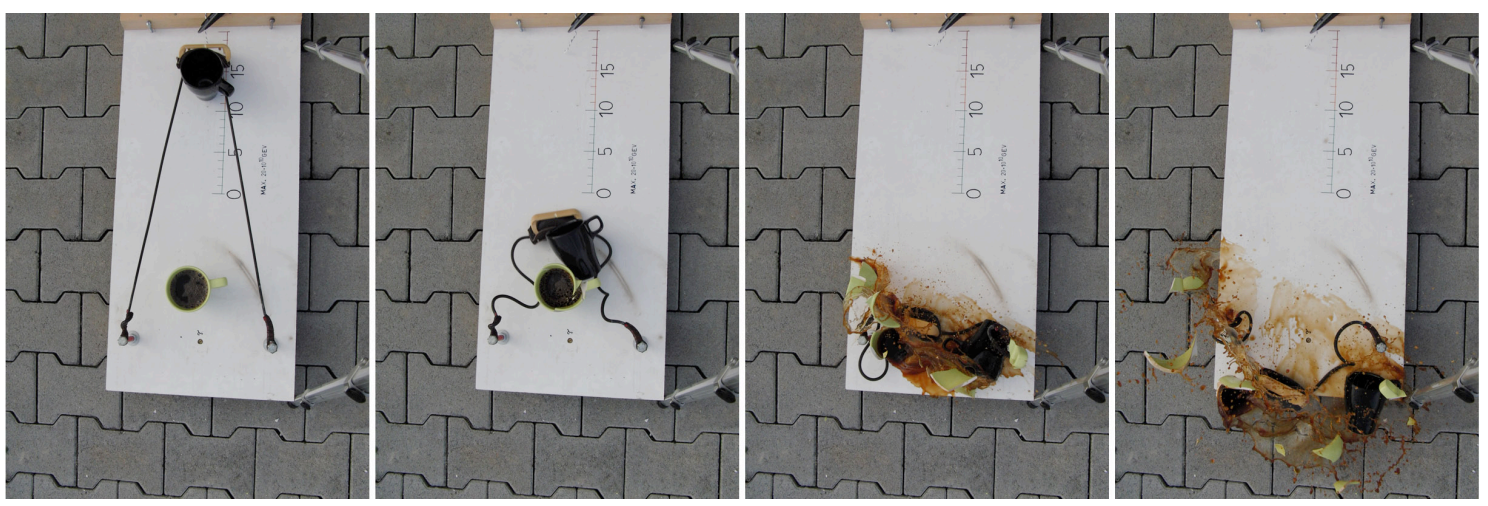

Figure 3: Collision of coffee cups. The image sequence was taken with $1 / 15 \mathrm{~s}$ between frames shown here.

\section{Percolation}

The percolation model [9-15] is a model suitable for simulating fragmentation reactions without relying on assumptions of equilibrium thermodynamics, with a well-defined (and wellstudied) infinite size limit, and with the ability to investigate systems with extreme finite size corrections. There are in general bond- and site-percolation models, with the model introduced by us [8-10] being of the bond-percolation class, the sites representing the nucleons (a conserved quantity) and the bonds representing nearest neighbor interactions. The nuclear fragmentation event deposits energy, which breaks the bonds, resulting in isolated clusters of still-connected sites, which are identified as pre-fragments.

The main physics input is the connection between the probability to break a given bond and the nuclear reaction physics. If one uses a simple cubic lattice, for example, then each nucleon in bulk shares 6 bonds with its nearest neighbors. Since the binding energy per nucleon of nuclear matter is $15.75 \mathrm{MeV}$, this implies that it takes $5.25 \mathrm{MeV}$ on average to break each bond in this lattice. One can compute the breaking probability under different model assumptions, for example utilizing Glauber theory. For a thermalized system of Fermions, one can derive a relation between the temperature and the breaking probability. Li et al. [16] developed the formalism to accomplish just this, a generalization of the formalism of Coniglio and Klein [17] for Fermion systems; and the result is

$$
p_{b}(T)=1-2 \Gamma\left(\frac{3}{2}, 0, B / T\right) / \sqrt{\pi} .
$$

Here $T$ is the temperature, $B$ is the binding energy per nucleon, and $\Gamma$ is the generalized incomplete gamma function. The left panel of Figure 4 shows the comparison of the results of our model (histogram) to the fragment spectrum obtained by Bujak et al. [18] for the reaction of $300 \mathrm{GeV}$ protons on a silver target (circles), which they conducted at Fermilab. 
This approach does not just work in nuclear physics, but also in the fragmentation of other systems. On the right side of Figure 4 we show the comparison of our percolation model (histogram) with the data obtained for the fragmentation of buckyball molecules $\left(\mathrm{C}_{60}\right)$ by xenon ions accelerated to a total kinetic energy of $625 \mathrm{MeV}$ [19-21]. In this system the energy deposition proceeds predominantly via electromagnetic excitation.

One can see that these two fragmenting systems have many features in common, despite the vastly different energy and size scales. First, the fragment distributions show a pronounced U-shape, which is due to the integration over different impact parameter bins and with it different excitation energy regions. Second there is a power law fall-off for low-to-intermediate mass fragments in both cases. The slower fall-off for the buckyballs $\left(\tau_{\text {eff }}=1.3\right)$ vs. that of the silver fragmentation ( $\tau_{\text {eff }}=2.6$ ) can be explained as a result of the different topologies of the molecules (surface of a sphere) vs. that of the nuclei (solid sphere). Finally, there are quantum mechanical binding energy effects superimposed in the power-laws in both cases.

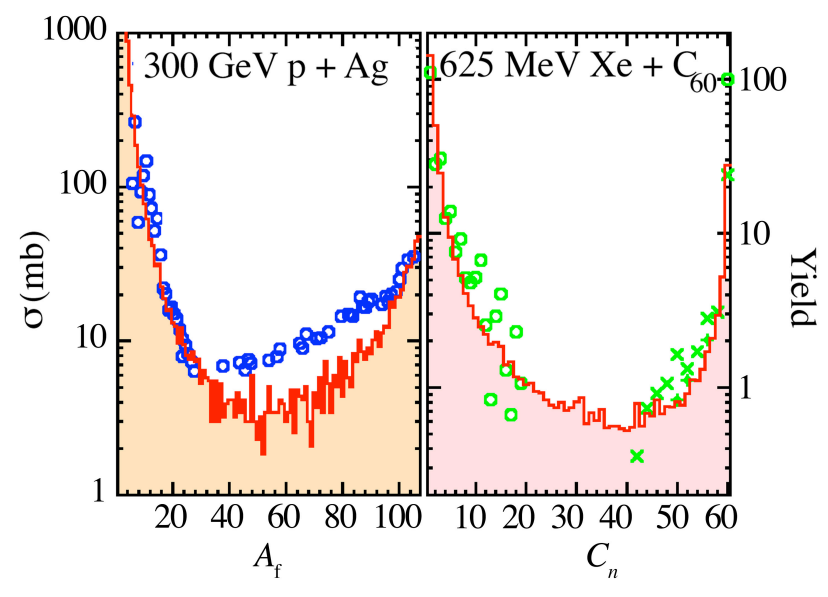

Figure 4: Fragment distributions from fragmentation reactions of nuclei (left) and buckyball molecules (right). Histograms: calculations with percolation-based models; symbols: data.

\subsection{Universality Class Determination}

An event-by-event moment analysis of fragmentation events near the critical point enables us to extract information on the universality class of the phase transition $[14,15]$. It is also possible to bin the data according to fragment multiplicity in a given event and then to perform a moment analysis as a function of the multiplicity. This is done in Figure 5 for the reaction of $10.8 \mathrm{GeV}$ pions colliding with a gold target. The parameter free comparison between the percolation theory calculations (histograms) and the data [22,23] (circles and squares) is shown for the two cases of including all fragments (upper curves and data points) and excluding the largest fragment (lower curves and data points). The agreement of the calculations [24,25] is a clear indication that the percolation model captures the essential features of the fragmentation process. This is in agreement with earlier calculations [26] performed for the fragmentation of gold projectiles on carbon targets in reverse kinematics [27,28]. 
The universality class of a phase transition can be determined by measuring the values of the critical exponents. From renormalization group results, we know that these critical exponents obey scaling relations, $v d=2-\alpha=2 \beta+\gamma=\beta(\delta+1)$. This implies that knowledge of two critical exponents (for example $\beta$ and $\delta$ ) is sufficient to determine the universality class.

Finally, a scaling analysis with a minimum $\chi^{2}$ parameter optimization performed in [24,25] yields the value of the critical exponents $\sigma=0.5 \pm 0.1$ and $\tau=2.35 \pm 0.05$, as well as the critical temperature of $T_{c}=(8.3 \pm 0.2) \mathrm{MeV}$ for the pion induced fragmentation data of gold. This result reflects the extreme finite size modifications of the obtained parameters for the system under consideration.

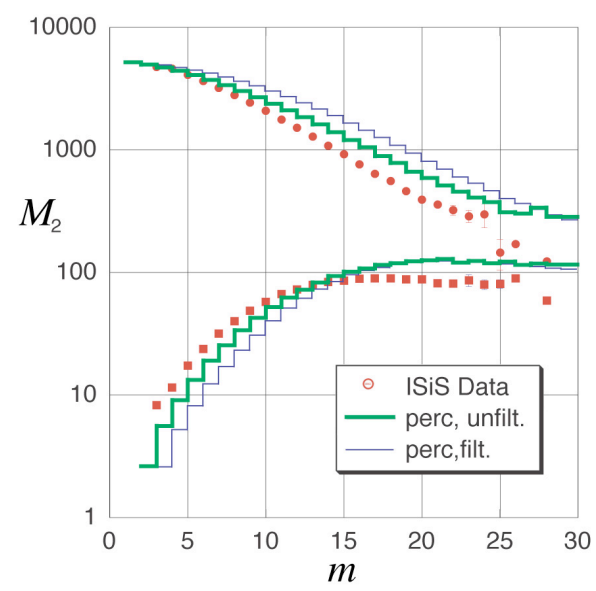

Figure 5: Second moment of the fragment distributions as a function of total fragment multiplicity for the reaction of $10.8 \mathrm{GeV}$ pions on gold.

\section{Conclusion}

The nuclear fragmentation problem has yielded a surprising amount of information during the last few decades, and it has proven to be a wonderful playground for many ideas of many-body physics from different fields. In particular the application of percolation theory has yielded excellent agreement with available data on fragment spectra, moments, multiplicities, and other observables. The picture of fragmentation as a non-equilibrium phase transition of the percolation kind is an attractive one, and it is supported by experimental evidence. What was introduced 27 years ago at the $23^{\text {rd }}$ Bormio meeting has stood the test of time.

\section{References}

[1] W. Bauer and G.D. Westfall, University Physics, McGraw-Hill (2011).

[2] G. Sauer, H. Chandra and U. Mosel, Nucl. Phys. A264 (1976) 221. 
[3] J.Pochodzalla et al. (ALADIN), Phys. Rev. Lett. 75, (1995) 1040.

[4] A.S. Hirsch et al., Phys. Rev. C 29 (1984) 508.

[5] P. Bak, C. Tang, and K. Wiesenfeld, Phys. Rev. Lett. 59, (1987) 381.

[6] P. Bak and K. Chen, Sci. Am. 264(1) (1991) 46.

[7] W. Bauer and S. Pratt, Phys. Rev. E 54 (1996) R1009.

[8] W. Bauer, D.R. Dean, U. Mosel, and U. Post, Multi-Fragmentation Reactions Within the Nuclear Lattice Model, Proceedings of the XXIII International Winter Meeting on Nuclear Physics, Bormio, Italy, (1985) 432.

[9] W. Bauer et al., Phys. Lett. B150 (1985) 53.

[10] W. Bauer et al., Nucl. Phys. A452 (1986) 699.

[11] T. Biro et al., Nucl. Phys. A459 (1986) 692.

[12] J. Nemeth et al., Z. Phys. A325 (1986) 347.

[13] J. Desbois, Nucl. Phys. A466 (1987) 724.

[14] X. Campi, J. Phys. A19 (186) L917.

[15] W. Bauer, Phys. Rev. C 38 (1988) 1927.

[16] T. Li et al., Phys. Rev. Lett. 70 (1993) 1924.

[17] A. Coniglio and A. Klein, J. Phys. A13 (1980) 2775.

[18]A. Bujak et al., Phys. Rev. C 32 (1985) 620.

[19] T. LeBrun et al., Phys. Rev. Lett. 72 (1994) 3965.

[20] R. Ali et al., Nucl. Inst. Meth. B 96 (1995) 479.

[21] S. Cheng et al., Phys. Rev. A 54 (1996) 3182.

[22] T. Lefort et al., Phys. Rev. Lett. 83 (1999) 4033.

[23] L. Beaulieu et al., Phys. Lett. B163 (1999) 159.

[24] W. Bauer et al., Heavy Ion Physics 15 (2001) 217.

[25] M. Kleine Berkenbusch et al., Phys. Rev. Lett. 88, (2002) 022701.

[26] W. Bauer and A. Botvina, Phys. Rev. C 52, (1995) R1760.

[27] M.L. Giles et al., Phys. Rev. Lett. 73 (1994) 1590.

[28] H.G. Ritter et al., Nucl. Phys. A507 (1995) 419c. 